\title{
Relation between bulk order-parameter correlation function and finite-size scaling
}

\author{
X.S. Chen ${ }^{1,2, a}$ and V. Dohm ${ }^{1, b}$ \\ ${ }^{1}$ Institut für Theoretische Physik, Technische Hochschule Aachen, \\ D-52056 Aachen, Germany \\ ${ }^{2}$ Institute of Particle Physics, Hua-Zhong Normal University, \\ Wuhan 430079, P.R. China
}

January 1, 2018

\begin{abstract}
We study the large-distance behavior of the bulk order-parameter correlation function $G(\mathbf{r})$ for $T>T_{c}$ within the lattice version of the $\varphi^{4}$ theory including lattice effects. We also study the large- $L$ behavior of the susceptibility $\chi$ for $T>T_{c}$ of the confined lattice system of linear size $L$ with periodic boundary conditions. We find that the structure of the large- $L$ behavior of $\chi$ of the confined system is closely related to the structure of the large-distance behavior of $G(\mathbf{r})$ of the bulk system. Explicit results are derived in the spherical (large- $n$ ) limit and in oneloop order for general dimensions $d>2$. For the lattice model with cubic symmetry we find that finite-size scaling must be formulated in terms of the anisotropic bulk correlation length (exponential correlation length) that governs the exponential decay of $G(\mathbf{r})$ for large $r$ rather than in terms of the ordinary isotropic bulk correlation length $\xi$ defined via the second moment of $G(\mathbf{r})$. We show that it is the exponential bulk correlation length $\xi_{1}$ in the direction of the cubic axes
\end{abstract}


that determines the exponential finite-size scaling behavior of lattice systems in a rectangular geometry. This result modifies a recent interpretation concerning an apparent violation of finite-size scaling in terms of the second-moment correlation length $\xi \neq \xi_{1}$. Exact results for the one-dimensional Ising model illustrate our conclusions. Furthermore we show for general $d>2$ that a description of finite-size effects for finite $n$ in the entire region $0 \leq L / \xi \leq \infty$ requires two different perturbative approaches that are applicable either to the region $0 \leq L / \xi \lesssim O(1)$ or $O(1) \lesssim L / \xi \leq \infty$, respectively. In particular we show that the exponential finite-size behavior for $L / \xi \gg 1$ above $T_{c}$ is not captured by the standard perturbation approach that separates the homogeneous lowest mode from the inhomogeneous higher modes. Consequences for the theory of finite-size effects above four dimensions are discussed. We show that the two-variable finite-size scaling form predicts an exponential approach $\propto e^{-L / \xi_{1}}$ to the bulk critical behavior above $T_{c}$ whereas the reduction to a single-variable scaling form implies a power-law approach $\propto L^{-d}$.

PACS: 05.70.Jk, 64.60.-i

${ }^{a}$ e-mail: chen@physik.rwth-aachen.de

${ }^{b}$ e-mail: vdohm@physik.rwth-aachen.de 


\section{Introduction}

The fundamental length scale near a critical point is the bulk correlation length $\xi$ which is a measure of the range of correlations of the order-parameter fluctuations. In this paper we consider lattice systems with cubic symmetry at and above $T_{c}$. We assume short-range interactions. The bulk orderparameter correlation function $G(\mathbf{r})$ between two lattice points at relative separation $\mathbf{r}$ serves to define $\xi$ via the second moment of $G(\mathbf{r})$ according to

$$
\xi^{2}=\sum_{\mathbf{r}} r^{2} G(\mathbf{r}) / \sum_{\mathbf{r}} G(\mathbf{r})
$$

In terms of the Fourier transform $\hat{G}(\mathbf{k})$ this definition reads

$$
\xi^{2}=\left.\hat{G}(\mathbf{0}) \frac{\partial}{\partial k^{2}}[\hat{G}(\mathbf{k})]^{-1}\right|_{k=0} .
$$

This is applicable to lattice systems with cubic symmetry whose correlation

function $\hat{G}(\mathbf{k})$ has an isotropic small $\mathbf{k}$ behavior at $O\left(k^{2}\right)$. The "second moment correlation length" (1.2) is widely used in field-theoretic calculations [1, 2, 3] since $\xi$ is identical with the inverse mass $m^{-1}=\xi$ that enters the two-point vertex function $\Gamma^{(2)}(\mathbf{k})=\hat{G}(\mathbf{k})^{-1}$.

The bulk correlation length (1.2) plays a fundamental role also in the formulation of the finite-size scaling behavior of confined systems. Consider, for example, the susceptibility $\chi(t, L) \propto \hat{G}(\mathbf{0})$ of a ferromagnetic system for $t=\left(T-T_{c}\right) / T_{c}>0$ in a finite geometry with a characteristic size $\mathrm{L}$ and with periodic boundary conditions. It is believed that the relative deviation from bulk critical behavior $\chi(t, \infty)=A_{\chi} t^{-\gamma}$ has the asymptotic (large $L$, small $t$ ) scaling form [4, 5, 6, 7, 8] below four dimensions 


$$
\Delta \chi=\frac{\chi(t, \infty)-\chi(t, L)}{\chi(t, \infty)}=g(L / \xi)
$$

where $g(x)$ is a universal function in the entire range $0 \leq x \leq \infty$. This means that any dependence of $\Delta \chi$ on the lattice constant $\tilde{a}$ is negligible in the asymptotic region $\xi \gg \tilde{a}, L \gg \tilde{a}$ for arbitrary ratios $L / \xi$, including the large- $L$ behavior at fixed $\xi \gg \tilde{a}[4,9]$. In a recent paper we have shown [10] that this statement is not valid in the regime $L / \xi \gg 1$. Specifically, for cubic geometry of size $L \gg \xi \gg \tilde{a}$, we have found

$$
\Delta \chi=g(L / \xi) \exp \left\{\Gamma(\tilde{a} / \xi) \frac{L}{\xi}\right\} .
$$

Here $g(x)$ is indeed universal but the $\tilde{a}$ dependent exponential factor with the nonuniversal function

$$
\Gamma(\tilde{a} / \xi)=\frac{1}{24}(\tilde{a} / \xi)^{2}+O\left[(\tilde{a} / \xi)^{3}\right]
$$

is non-negligible for $L \gtrsim 24 \xi^{3} / \tilde{a}^{2}$, i.e., for sufficiently large $L$ close to the bulk limit above $T_{c}$, even in the asymptotic region $\xi \gg \tilde{a}$. Thus the finite-size scaling form (1.3) in terms of the second-moment correlation length (1.2) is not valid for lattice systems, even below four dimensions. This conclusion is based on one-loop results as well as on exact results in the spherical limit of the $\varphi^{4}$ lattice model at finite lattice spacing [10]. We note that the possibility 
of a non-negligible dependence of finite-size effects on the lattice spacing $\tilde{a}$, even for $d<4$, was already mentioned by Privman and Fisher [11]. An as yet unexplained $\tilde{a}$-dependence that is not consistent with a finite-size scaling ansatz of the kind (1.3) was also found by Gelfand and Fisher [12] in the interfacial free energy of the two-dimensional Ising model for $T<T_{c}$.

No intuitive reasoning was given in our recent work [10] as to what might be the physical origin for this unexpected failure of the finite-size scaling property. It is the purpose of the present paper to elucidate this unsatisfactory situation by further analyzing the role played by the correlation length in both bulk and confined lattice systems. Instead of the second-moment correlation length (1.2) we consider the "exponential correlation length" $\xi_{\mathbf{e}}$ that governs the large-distance behavior of the bulk system at fixed $T$ above $T_{c}$ [13,

$$
G(r \mathbf{e}) \sim B_{d} r^{(1-d) / 2} \exp \left(-r / \xi_{\mathbf{e}}\right),
$$

where $\mathbf{e}=\mathbf{r} / r$ is the unit vector in the direction of $\mathbf{r}$ and where $\xi_{\mathbf{e}}$ is defined by

$$
\xi_{\mathbf{e}}^{-1}=-\lim _{r \rightarrow \infty}\{[\ln G(r \mathbf{e})] / r\} .
$$

We note that (1.6) is expected to hold even well above $T_{c}$ outside the asymptotic critical region. For lattice systems, $\xi_{\mathbf{e}}$ is an anisotropic quantity. It is 
expected, however, that the asymptotic ratio $\lim _{t \rightarrow 0+} \xi_{\mathbf{e}} / \xi$ becomes isotropic and has a universal value [14, 15, 16, 17, 18], in agreement with our results.

In the present paper we shall call attention to the fact that the non-universal difference between $\xi$ and $\xi_{\mathbf{e}}$ is non-negligible even asymptotically close to $T_{c}$ if the scaling function has an exponential form. We shall show that lattice effects cause additive non-universal corrections to the asymptotic form of $\xi_{\mathbf{e}}$ [see (1.9) below]. Since $\xi_{\mathbf{e}}$ appears in the exponent of the scaling functions these additive corrections turn into non-negligible multiplicative overall factors for the exponential scaling functions of both $G(\mathbf{r})$ for $r \gg \xi_{\mathbf{e}}$ and $\chi(t, L)$ for $L \gg \xi_{\mathbf{e}}$.

Because of the anisotropy of $\xi_{\mathbf{e}}$ for lattice systems, there exist infinitely many $\xi_{\mathbf{e}}$ in contrast to the unique isotropic quantity $\xi$. Since $\chi(t, L) \propto \hat{G}(\mathbf{0}) \propto$ $\sum_{\mathbf{r}} G(\mathbf{r})$ involves all directions of $\mathbf{r}$ it is not clear a priori whether a certain average of $\xi_{\mathbf{e}}$ or $\xi_{\mathbf{e}}$ in a particular direction e enters the finite-size scaling form of $\chi(t, L)$. Here we shall find that for cubic geometry the exponential finite-size effect is determined by the correlation length $\xi_{1} \equiv \xi_{\mathbf{e}_{1}}$ (and not by $\left.\xi \neq \xi_{1}\right)$ where $\mathbf{e}_{1}$ is the unit vector in the direction of one of the cubic axes. Specifically we derive an explicit relation between $\xi_{1}$ and $\xi$ in the spherical limit (Sect. 3) and in one-loop order of the $\varphi^{4}$ lattice model (Sect. 4). This relation reads at finite lattice spacing $\tilde{a}$

$$
\xi=\frac{\tilde{a}}{2}\left[\sinh \left(\frac{\tilde{a}}{2 \xi_{1}}\right)\right]^{-1}
$$




$$
=\xi_{1}\left[1-\frac{1}{24}\left(\frac{\tilde{a}}{\xi_{1}}\right)^{2}+\ldots\right] .
$$

It is remarkable that the non-universal $\tilde{a}$ dependence of (1.4) is completely absorbed by the exponential correlation length $\xi_{1}$ if the function $\xi\left(\xi_{1}\right)$ of $(1.8)$ is substituted into the right-hand side of (1.4). In the present paper we shall show that for $L \gg \xi \gg \tilde{a}$

$$
g\left(L / \xi\left(\xi_{1}\right)\right) \exp \left\{\Gamma\left(\tilde{a} / \xi\left(\xi_{1}\right)\right) \frac{L}{\xi\left(\xi_{1}\right)}\right\}=g\left(L / \xi_{1}\right)
$$

hence universal finite-size scaling below four dimensions at finite lattice spacing is restored in the form

$$
\Delta \chi=g\left(L / \xi_{1}\right)
$$

with the universal function $g(x)$.

Thus, at one-loop order and in the spherical (large- $n$ ) limit, there is no violation of finite-size scaling at finite lattice spacing below four dimensions provided that the exponential correlation length $\xi_{1}$ rather than $\xi$ is employed as the bulk reference length. We note that this result is not a general consequence of the renormalizability of the $\varphi^{4}$ theory but is only an explicit computational result for cubic (and rectangular) geometry in one-loop order 
and in the spherical (large- $n$ ) limit. It is also valid for the one-dimensional Ising model (Sect. 6). At present it is an open question whether this result remains valid at finite $n$ beyond one-loop order and whether it can be based on more general arguments. The renormalization-group (RG) arguments in the limit $\tilde{a} \rightarrow 0$ [國] are not sufficient to establish the complete finite-size scaling form for lattice systems. Although these RG arguments for $\tilde{a} \rightarrow 0$ lead to the same universal scaling function $g(x)$ as our analysis at finite $\tilde{a}$, they do not identify the $\tilde{a}$ dependent finite-size scaling variable $x=L / \xi_{1}$.

We shall also show (Sect. 4) that two different perturbative treatments are necessary to describe the finite-size effects in the entire asymptotic region $0 \leq L / \xi \leq \infty$ and that the previous finite-size perturbation approach below four dimensions [19, 20, 21] does not capture the exponential structure of the scaling function $g(x) \propto e^{-x}$ for $x \gg 1$.

The necessity of employing $\xi_{1}$ rather than $\xi$ is not restricted to $d<4$ dimensions. In Sect. 5 we shall discuss the consequences of our results for lattice systems with $d>4$ where $\xi_{1}$ rather than $\xi$ should be incorporated in the two-variable finite-size scaling form [22, 23]. The inhomogeneous modes are shown to yield $\Delta \chi \propto e^{-L / \xi_{1}}$ for $L \gg \xi$ whereas the lowest-mode approximation [19] and the corresponding single-variable finite-size scaling form [24] imply $\Delta \chi \propto L^{-d}$.

Very recently an exponential size dependence has been confirmed by Stauffer [25] in Monte Carlo simulations for the magnetization of the Ising model in two, three and five dimensions [26]. 
In summary, even though we will confirm through (1.9) (within our approximations) that asymptotically close to the critical point the two correlation lengths $\xi$ and $\xi_{1}$ are the same and isotropic, the scaling form $g(x)$ of the leading finite-size effect near the bulk limit is sensitive to the choice of the correlation length because of the exponential dependence of $g(x) \propto e^{-x}$ on the correlation length. 


\section{Lattice effect on the large-distance behav- ior of the bulk order-parameter correlation function}

We consider a $\varphi^{4}$ lattice Hamiltonian for the variables $\varphi_{i}$ on the lattice points $\mathbf{x}_{i}$ of a simple-cubic lattice with the lattice constant $\tilde{a}$. The variables $\varphi_{i}$ have $n$ components $\varphi_{i \alpha}$ with $\alpha=1,2, \ldots, n$ which vary in the range $-\infty \leq \varphi_{i \alpha} \leq$ $\infty$. We assume the statistical weight $\propto e^{-H}$ with

$$
H=\tilde{a}^{d}\left\{\sum_{i}\left[\frac{r_{0}}{2} \varphi_{i}^{2}+u_{0}\left(\varphi_{i}^{2}\right)^{2}\right]+\sum_{i, j} \frac{1}{2 \tilde{a}^{2}} J_{i j}\left(\varphi_{i}-\varphi_{j}\right)^{2}\right\}
$$

where $J_{i j}$ are the dimensionless couplings of a short-rang interaction with cubic symmetry and where $k_{B} T \approx k_{B} T_{c}$ is absorbed in $r_{0}, u_{0}$ and $J_{i j}$. The

variables $\varphi_{i}$ have the dimension $\left[\tilde{a}^{(2-d) / 2}\right]$. We are interested in the largedistance behavior of the bulk correlation function

$$
G\left(\mathbf{x}_{j}-\mathbf{x}_{0}\right)=\frac{1}{n}\left\langle\varphi_{j} \varphi_{0}\right\rangle=\int_{\mathbf{k}} \hat{G}(\mathbf{k}) e^{i \mathbf{k}\left(\mathbf{x}_{j}-\mathbf{x}_{0}\right)}
$$

above $T_{c}$, normalized to the number of components $n$, where

$$
\hat{G}(\mathbf{k})=\frac{\tilde{a}^{d}}{n} \sum_{j}\left\langle\varphi_{j} \varphi_{0}\right\rangle e^{-i \mathbf{k}\left(\mathbf{x}_{j}-\mathbf{x}_{0}\right)}
$$


with some fixed lattice point $\mathbf{x}_{0}$. In (2.2) $\int_{\mathbf{k}}$ stands for $(2 \pi)^{-d} \int d^{d} k$ with a finite lattice-cutoff $\left|k_{m}\right| \leq \pi / \tilde{a}, m=1,2, \ldots, d$. First we consider the limit $n \rightarrow \infty$ at fixed $u_{0} n$ for $d>2$ in which case we obtain [27]

$$
\begin{gathered}
\hat{G}(\mathbf{k})^{-1}=\hat{G}(\mathbf{0})^{-1}+\hat{J}_{\mathbf{k}}, \\
\hat{J}_{\mathbf{k}}=\frac{2}{\tilde{a}^{2}}[J(\mathbf{0})-J(\mathbf{k})], \\
J(\mathbf{k})=(\tilde{a} / L)^{d} \sum_{i, j} J_{i j} e^{-i \mathbf{k}\left(\mathbf{x}_{j}-\mathbf{x}_{i}\right)} .
\end{gathered}
$$

$\hat{G}(\mathbf{0})$ is determined by an implicit equation [27] which, however, will not be needed in the following since $\hat{G}(\mathbf{0})$ can be expressed directly in terms of $\xi^{2}$. Using the second-moment definition for the bulk correlation length $\xi$ according to (1.2) we have 27

$$
\begin{gathered}
\hat{G}(\mathbf{0})=J_{0}^{-1} \xi^{2}, \\
J_{0}=\frac{1}{d}(\tilde{a} / L)^{d} \sum_{i, j}\left(J_{i j} / \tilde{a}^{2}\right)\left(\mathbf{x}_{i}-\mathbf{x}_{j}\right)^{2} .
\end{gathered}
$$


For simplicity we consider a nearest-neighbor interaction $J>0$ which yields

$$
\begin{gathered}
J(\mathbf{k})=2 J \sum_{m=1}^{d} \cos \left(k_{m} \tilde{a}\right), \\
\hat{J}_{\mathbf{k}}=\frac{4 J}{\tilde{a}^{2}} \sum_{m=1}^{d}\left[1-\cos \left(k_{m} \tilde{a}\right)\right]=J_{0} \mathbf{k}^{2}+O\left(k_{i}^{2} k_{j}^{2}\right)
\end{gathered}
$$

with $J_{0}=2 J$.

In summary we need to calculate the large-distance behavior of

$$
G(\mathbf{x})=\frac{\tilde{a}^{2}}{2 J} \int_{\mathbf{k}} e^{i \mathbf{k x}}\left\{(\tilde{a} / \xi)^{2}+2 \sum_{m=1}^{d}\left[1-\cos \left(k_{m} \tilde{a}\right)\right]\right\}^{-1}
$$

where we have chosen $\mathbf{x}_{0}=\mathbf{0}$ and $\mathbf{x}_{j} \equiv \mathbf{x}=\left(x_{1}, x_{2}, \ldots, x_{d}\right)$ with Cartesian coordinates $x_{m}$. Eq. (2.11) is valid not only in the large- $n$ limit but also in an ordinary perturbation calculation to one-loop order for general $n$. (For the latter case a renormalization-group treatment is carried out in Appendix B for $d<4$.) A representation of $G(\mathbf{x})$ in terms of Bessel functions of integer order $\nu$ (see, e.g., 9.6.19 of Ref. [28])

$$
I_{\nu}(z)=\frac{1}{\pi} \int_{0}^{\pi} d \theta e^{z \cos \theta} \cos (\nu \theta)
$$


can be given as

$$
\begin{gathered}
G(\mathbf{x})=\frac{\tilde{a}^{2}}{2 J} \int_{0}^{\infty} d s e^{-(\tilde{a} / \xi)^{2} s} \int_{\mathbf{k}} e^{i \mathbf{k x}} \exp \left\{-2 s \sum_{m=1}^{d}\left[1-\cos \left(k_{m} \tilde{a}\right)\right]\right\} \\
=\frac{\tilde{a}^{2-d}}{2 J} \int_{0}^{\infty} d s e^{-(\tilde{a} / \xi)^{2} s} e^{-2 d s} \prod_{m=1}^{d} I_{\nu_{m}}(2 s)
\end{gathered}
$$

with the integers $\nu_{m}=x_{m} / \tilde{a}$. In general, $G(\mathbf{x})$ is an anisotropic function whose exponential large-distance behavior $\sim \exp \left(-|\mathbf{x}| / \xi_{\mathbf{e}}\right)$ leads to the definition of an anisotropic correlation length $\xi_{\mathbf{e}} \neq \xi$ in the direction of the unit vector $\mathbf{e}=\mathbf{x} /|\mathbf{x}|[13]$. An explicit demonstration of the anisotropy of $\xi_{\mathbf{e}}$ is given in Appendix A where the angular dependence of $\xi_{\mathbf{e}}$ is calculated for the case where e lies in the 2 -dimensional $x_{1}-x_{2}$ plane of the d-dimensional bulk system. For our present purpose it suffices to consider only the special case where $\mathbf{e}=\mathbf{e}_{1}=(1,0,0 \ldots)$ is the unit vector along one of the cubic axes. Then we have $\mathbf{x}=(x, 0,0 \ldots)$ and $\mathbf{k x}=k_{x} x$. The corresponding correlation function is denoted by $C(x)=G(\mathbf{x})$ which is obtained from (2.14) as

$$
C(x)=\frac{\tilde{a}^{2-d}}{2 J} \int_{0}^{\infty} d s e^{-(\tilde{a} / \xi)^{2} s} e^{-2 d s}\left[I_{0}(2 s)\right]^{d-1} I_{x / \tilde{a}}(2 s) .
$$

This result is valid for arbitrary $x / \tilde{a}$ and $\xi / \tilde{a}$ and therefore does not yet have a scaling form. In Appendix A the large- $|x|$ behavior of $C(x)$ at arbitrary fixed $\xi / \tilde{a}$ is derived. The result is 


$$
C(x)=\frac{\tilde{a}^{2-d}}{4 J}\left(\frac{\tilde{a}}{2 \pi|x|}\right)^{(d-1) / 2}\left[\sinh \left(\frac{\tilde{a}}{\xi_{1}}\right)\right]^{(d-3) / 2} e^{-|x| / \xi_{1}}\left[1+O\left(|x|^{-1}\right)\right]
$$

We see that in the large- $|x|$ limit the natural reference length is the exponential correlation length $\xi_{1} \equiv \xi_{\mathbf{e}_{1}}$ in the direction of one of the cubic axes rather than the second-moment correlation length $\xi$. The exact relation between $\xi_{1}$ and $\xi$ for $n \rightarrow \infty$ reads

$$
\xi^{-1}=\frac{2}{\tilde{a}} \sinh \left(\frac{\tilde{a}}{2 \xi_{1}}\right)
$$

or

$$
\xi_{1}^{-1}=\frac{2}{\tilde{a}} \operatorname{arsinh}\left(\frac{\tilde{a}}{2 \xi}\right)
$$

The difference between $\xi$ and $\xi_{1}$ is a true lattice effect that disappears in the formal limit $\tilde{a} \rightarrow 0$. Eqs. (2.17) and (2.18) are also valid for finite $n$ in one-loop order above $T_{c}$ but in two-loop order and beyond we expect (small) corrections to (2.17) and (2.18) for finite $n$.

Eqs.(2.16)-(2.18) are valid for arbitrary $\xi_{1} / \tilde{a}$ even well above $T_{c}$. In the asymptotic region $\xi_{1} \gg \tilde{a}$, 2.16) attains the scaling form 


$$
C(x) \sim(\tilde{a} /|x|)^{d-2+\eta} \Phi\left(|x| / \xi_{1}\right)
$$

with the scaling function for $|x| / \xi_{1} \gg 1$

$$
\Phi\left(|x| / \xi_{1}\right)=\tilde{A} \frac{\tilde{a}^{2-d}}{4 J(2 \pi)^{(d-1) / 2}}\left(\frac{|x|}{\xi_{1}}\right)^{\frac{1}{2}(d-3)+\eta} \exp \left(-|x| / \xi_{1}\right)
$$

where $\eta=0$ and $\tilde{A}=1$ in the present case of the limit $n \rightarrow \infty$. Eqs. (2.19) and (2.20) are also valid for general $n$ in one-loop order for $d<4$, see Appendix B. In this case we have a critical exponent $\eta>0$ and an amplitude $\tilde{A} \neq 1$ which we obtain from a RG treatment at finite $\tilde{a}$ [10, applied to the bare one-loop result (2.11) for general $n$, as described in Appendix B. Eqs. (2.19) and (2.20) are also valid in one-loop order for general $n$ and $d>4$ where $\eta=0$ and $\tilde{A}=1$, apart from $O\left(u_{0}^{2}\right)$ corrections.

Close to $T_{c}$ where both $\xi$ and $\xi_{1}$ diverge, an expansion of (2.18) yields

$$
\xi_{1}=\xi\left[1+\frac{1}{24}\left(\frac{\tilde{a}}{\xi}\right)^{2}+\ldots\right]
$$

Thus, for $n \rightarrow \infty, \xi_{1}$ and $\xi$ become identical sufficiently close to $T_{c}$. (This is also valid for finite $n$ in one-loop order above $T_{c}$ but in two-loop order and beyond the asymptotic value of the ratio $\xi_{1} / \xi$ for $T \rightarrow T_{c}$ is expected to become different from 1, see e.g. Refs.[14, 15, 16, 17, 18].) Therefore $\xi_{1}$ can 
be replaced by $\xi$ in the prefactor of (2.20). We emphasize, however, that a replacement of $\xi_{1}$ by $\xi$ is not possible in the exponential part of (2.16) and (2.20), even arbitrarily close to $T_{c}$. This is seen by substituting (2.21) into the exponential function of (2.16) and (2.20),

$$
\begin{gathered}
e^{-|x| / \xi_{1}}=\exp \left(|x| \tilde{a}^{2} / 24 \xi^{3}\right) e^{-|x| / \xi}, \\
C(x) \sim(\tilde{a} /|x|)^{d-2+\eta} \Phi(|x| / \xi) \exp \left(|x| \tilde{a}^{2} / 24 \xi^{3}\right) .
\end{gathered}
$$

Now the additive correction in (2.21) has turned into an exponential nonuniversal prefactor in (2.22) and (2.23) that cannot be simply replaced by 1 and that is by no means negligible for sufficiently large $|x| \gtrsim 24 \xi^{3} / \tilde{a}^{2}$, even in the asymptotic critical region $\xi \gg \tilde{a}$. This is the crucial point of our argument.

Thus, in order to have a universal ( $\tilde{a}$ independent) scaling form of $C(x)$ for large $|x| \gg \xi$ at fixed $T$ above $T_{c}$ where $C(x)$ has an exponential form, it is inescapable to employ $\xi_{1}$ rather than $\xi$ as the appropriate reference length. Correspondingly, for any $T>T_{c}$, there exists an infinitely large region $|x| \gtrsim 24 \xi^{3} / \tilde{a}^{2}$ where the anisotropy of $G(\mathbf{x})$ is no longer a negligible correction to the isotropic part. In the critical region $\tilde{a} \ll|\mathbf{x}| \ll \xi$, on the other hand, where $G(\mathbf{x})$ has a power-law behavior the nonuniversal part of the difference between $\xi$ and $\xi_{\mathbf{e}}$ can be considered as a negligible non-asymptotic 
additive correction. The natural reference lengths in these two regions are $\xi$ and $\xi_{\mathbf{e}}$, respectively, and a complete scaling description should embody a kind of crossover in the scaling variable from $|\mathbf{x}| / \xi$ to $\mathbf{x} / \xi_{\mathbf{e}}$. The same situation will arise in the finite-size problem with respect to the $L$ dependence of the susceptibility that is analyzed in the next Section.

The analysis of this Section can be extended to the continuum version of the $\varphi^{4}$ theory. In a separate paper [29] we shall show that the results depend on the cutoff procedure. An (isotropic) exponential large- $|\mathbf{x}|$ behavior of $G(\mathbf{x})$ is found for a smooth cutoff whereas a sharp cutoff implies a nonuniversal non-exponential $|\mathbf{x}|$-dependence of $G(\mathbf{x})$. In the case of a smooth cutoff it is also found that the exponential correlation length differs from the secondmoment correlation length even though the continuum system is isotropic. 


\section{Lattice effect on the finite-size scaling be- havior for $n \rightarrow \infty$}

We consider the lattice Hamiltonian (2.1) for a finite hypercubic geometry with volume $V=L^{d}$ and with periodic boundary conditions. We are interested in the exact large- $L$ behavior of the susceptibility $\chi=\hat{G}(\mathbf{0})$ above $T_{c}$ in the large- $n$ limit at finite lattice spacing $\tilde{a}$. Specifically we wish to identify the reference length that governs the expected exponential $L$-dependence at fixed $T>T_{c}$. The answer is not clear a priori since in the sum

$$
\chi=\frac{\tilde{a}^{d}}{n} \sum_{j}<\varphi_{j} \varphi_{0}>
$$

there are contributions from $<\varphi_{j} \varphi_{0}>$ in all directions involving all anisotropic correlation lengths $\xi_{\mathbf{e}}$ discussed in the preceding Section. The finite-size effect on $\chi$ at finite lattice constant $\tilde{a}$ has already been calculated previously [10, 22, 27] where it was expressed in terms of the second-moment correlation length $\xi$. Here we shall demonstrate that $\xi_{1}$ as calculated in the preceding Section, rather than $\xi$, is the appropriate reference length in the finite-size scaling structure.

We start from the implicit equation for $n \rightarrow \infty$ at fixed $u_{0} n$ [27]

$$
\chi^{-1}=r_{0}+4 u_{0} n L^{-d} \sum_{\mathbf{k}}\left(\hat{J}_{\mathbf{k}}+\chi^{-1}\right)^{-1}
$$


which can be rewritten as

$$
\begin{gathered}
\chi^{-1}=r_{0}-r_{0 c}+4 u_{0} n D\left(\chi^{-1}, L, \tilde{a}\right) \\
-4 u_{0} n \chi^{-1} \int_{\mathbf{k}}\left[\hat{J}_{\mathbf{k}}\left(\hat{J}_{\mathbf{k}}+\chi^{-1}\right)\right]^{-1}
\end{gathered}
$$

where $r_{0 c}=-4 u_{0} n \int_{\mathbf{k}} \hat{J}_{\mathbf{k}}^{-1}$. The finite-size effect is contained in the function

$$
\begin{gathered}
D\left(\chi^{-1}, L, \tilde{a}\right)=L^{-d} \sum_{\mathbf{k}}\left(\hat{J}_{\mathbf{k}}+\chi^{-1}\right)^{-1}-\int_{\mathbf{k}}\left(\hat{J}_{\mathbf{k}}+\chi^{-1}\right)^{-1} \\
\quad=\int_{0}^{\infty} d \tilde{s} e^{-\tilde{s} / \chi}\left\{L^{-d} \sum_{\mathbf{k}} e^{-\tilde{s} \hat{J}_{\mathbf{k}}}-\int_{\mathbf{k}} e^{-\tilde{s} \hat{J}_{\mathbf{k}}}\right\} .
\end{gathered}
$$

The summations run over discrete $\mathbf{k}$ vectors with components $k_{j}=2 \pi m_{j} / L, m_{j}=$ $0, \pm 1, \pm 2, \ldots, j=1,2, \ldots, d$, in the range $-\pi / \tilde{a} \leq k_{j}<\pi / \tilde{a}$. Since $\hat{J}_{\mathbf{k}}$ is a periodic function of each component $k_{j}$ the sum in (3.5) satisfies the Poisson identity [5, 30]

$$
L^{-d} \sum_{\mathbf{k}} e^{-\tilde{s} \hat{J}_{\mathbf{k}}}=\sum_{\mathbf{n}} \int_{\mathbf{k}} e^{-\tilde{s} \hat{J}_{\mathbf{k}}} e^{i \mathbf{k n} L}
$$

where $\mathbf{k} \cdot \mathbf{n}=\sum_{j} k_{j} n_{j}$. The sum $\sum_{\mathbf{n}}$ runs over all integers $n_{j}, j=1,2, \ldots, d$ in the range $-\infty \leq n_{j} \leq \infty$ whereas $\sum_{\mathrm{k}}$ and $\int_{\mathrm{k}}$ have finite cutoffs $\pm \pi / \tilde{a}$. For the case of a nearest-neighbor coupling $J>0$ we have 


$$
e^{-\tilde{s} \hat{J}_{\mathbf{k}}}=\prod_{m=1}^{d} \exp \left\{-\frac{4 J \tilde{s}}{\tilde{a}^{2}}\left[1-\cos \left(k_{m} \tilde{a}\right)\right]\right\}
$$

This leads to the representation in terms of the Bessel functions $I_{\nu}(z)$, (2.12),

$$
\begin{gathered}
\int_{\mathbf{k}} e^{-\tilde{s} \hat{J}_{\mathbf{k}}}=\left[\tilde{a}^{-1} e^{-2 s} I_{0}(2 s)\right]^{d} \\
L^{-d} \sum_{\mathbf{k}} e^{-\tilde{s} \hat{J}_{\mathbf{k}}}=\left[\tilde{a}^{-1} \sum_{n=-\infty}^{\infty} e^{-2 s} I_{\nu_{n}}(2 s)\right]^{d}
\end{gathered}
$$

where $s=2 \tilde{s} J / \tilde{a}^{2}$ and where $\nu_{n}=n L / \tilde{a}$ are integers. The resulting expression for $D$ reads

$$
\begin{aligned}
D\left(\chi^{-1}, L, \tilde{a}\right) & =\frac{\tilde{a}^{2-d}}{2 J} \int_{0}^{\infty} d s e^{-\tilde{a}^{2} s /(2 J \chi)} e^{-2 d s} \\
& \times\left\{\left[I_{0}(2 s)+\sum_{n=1}^{\infty} 2 I_{\nu_{n}}(2 s)\right]^{d}-\left[I_{0}(2 s)\right]^{d}\right\} .
\end{aligned}
$$

The large- $L$ limit corresponds to large integers $\nu_{n}=n L / \tilde{a}$. Since we consider this limit at fixed temperature above $T_{c}$ we may replace $\chi(t, L)$ in the exponent of (3.10) by the bulk value $\chi_{b}=\xi^{2} / 2 J$. For the asymptotic behavior of $I_{\nu}(2 s)$ we refer to Appendix A. The leading term for $L \gg \xi$ comes from the $n=1$ contribution in $(3.10)$, 


$$
D\left(\chi^{-1}, L, \tilde{a}\right)=\left(d \tilde{a}^{2-d} / J\right) \int_{0}^{\infty} d s e^{-(\tilde{a} / \xi)^{2} s} e^{-2 d s}\left[I_{0}(2 s)\right]^{d-1} I_{L / \tilde{a}}(2 s)
$$

This integral is identical with that of the bulk correlation function (2.15), except that the argument $x / \tilde{a}$ in $(2.15)$ is replaced here by $L / \tilde{a}$. Therefore the large- $L$ behavior of (3.11) is analogous to the large- $|x|$ behavior of $(2.16)$,

$D\left(\chi^{-1}, L, \tilde{a}\right)=\frac{d \tilde{a}^{2-d}}{2 J}(2 \pi L / \tilde{a})^{(1-d) / 2}\left[\sinh \left(\tilde{a} / \xi_{1}\right)\right]^{(d-3) / 2} e^{-L / \xi_{1}}\left[1+O\left(L^{-1}\right)\right]$

for $L \gg \xi_{1}$ where $\xi_{1}$ is the exponential bulk correlation length in the direction of one of the cubic axes as determined by (2.18). The exact parallelism between the large-distance behavior of the correlation function $C(x)$ and the large- $L$ behavior of the susceptibility $\chi(t, L)$ is the central result of this paper. On physical grounds it is quite plausible that for $L \gg \xi$ there exists a sensitivity of finite-size effects to the length $\xi_{1}$ governing the large-distance decay of $C(x)$ rather than to an averaged length as represented by the second moment $\xi$ of the correlation function.

The result (3.12) can be extended to a $d$-dimensional system with partially finite geometry that is confined in $\tilde{d}$ dimensions and infinite in $d-\tilde{d}$ dimensions. In this case the prefactor $d$ in (3.12) is replaced by $\tilde{d}$. For $\tilde{d}=1$ (film geometry) we find agreement with the result of Barber and Fisher [31, 32]. The authors did not recognize, however, that their quantity 
$\Gamma_{d}(T)=2 \operatorname{arsinh}\left(\Phi_{0}^{1 / 2} / 2\right)$ is identical with the inverse of the exponential bulk correlation length $\xi_{1} / \tilde{a}$.

The result (3.3) together with (3.12) is still valid for arbitrary $\xi_{1} / \tilde{a}$ even well above $T_{c}$. Using the known expression for the bulk susceptibility $\chi_{b}$ 10 we obtain from (3.3) and (3.12) the relative deviation from the bulk critical behavior for $L \gg \xi_{1} \gg \tilde{a}$ as

$$
\Delta \chi \equiv \frac{\chi_{b}-\chi}{\chi_{b}}=g\left(L / \xi_{1}\right)
$$

with the universal function in the large- $n$ limit for $2<d<4$

$$
g\left(L / \xi_{1}\right)=2 d \pi^{1 / 2}[\Gamma((4-d) / 2)]^{-1}\left(2 \xi_{1} / L\right)^{(d-1) / 2} e^{-L / \xi_{1}}
$$

This result agrees with and goes beyond our previous result in (132) - (134) or (135) of Ref. [10 which was expressed in terms of $\xi$ rather than $\xi_{1}$ (compare also (1.4) and (1.10) of the present paper). Previously we did not yet recognize the physical origin of the non-scaling contribution $R(L / \xi, \tilde{a} / \xi)$ in (134) of Ref. [10]. Now we see that $\xi_{\text {eff }}$ as defined in the paragraph after (107) of Ref. [10] turns out to be identical with $\xi_{1}$. This is parallel to the bulk order-parameter correlation function of Section 3. Thus our previous interpretation in terms of a violation of finite-size scaling below four dimensions in the region $L \gg \xi$ was incomplete for the lattice system (but not for the continuum system with a sharp cutoff [10, 29, 33]). In the critical 
region $\xi \gg L$, on the other hand, the natural reference length remains to be $\xi$ and not $\xi_{1}$. In this region the function $g(x)$ has a power-law form (not exponential) in which the non-universal part of the difference between $L / \xi_{1}$ and $L / \xi$ can be considered as a negligible correction.

We conclude that nonuniversal and non-negligible lattice effects do exist in the region $L \gg \xi$ but they can be absorbed in the finite-size scaling argument by employing the exponential bulk correlation length. This remedies the apparent violation of finite-size scaling found previously below four dimensions [10] and simplifies the physical picture of critical behavior in confined lattice systems with periodic boundary conditions. Nevertheless we maintain that a scaling description of the entire region $0 \leq L / \xi \leq \infty$ requires to embody in the scaling function a kind of crossover in the finite-size scaling variable from $x=L / \xi$ for $0 \leq x \lesssim O(1)$ to $x=L / \xi_{1}$ for $O(1) \leq x \leq \infty$.

So far our conclusions have only been shown to be correct in the spherical (large- $n$ ) limit for $2<d<4$ (and in one-loop order, see Section 4 , see also Section 6 for the $d=1$ Ising model). We note that Eq. (3.14) has a finite limit also for $d \rightarrow 2$ at fixed $\xi_{1}$. Further work is needed to prove whether finite-size scaling for lattice systems with periodic boundary conditions below four dimensions is indeed an asymptotically exact property for finite $n$ beyond one-loop order. General renormalization-group arguments [3, [] are not sufficient for such a proof, as shown in Sect. 3 of Ref. [10].

For a corresponding analysis of the large- $L$ behavior above $T_{c}$ within the continuum $\varphi^{4}$ theory we refer to Refs. [10, 29, 33]. In this case an exponential size dependence of $\Delta \chi$ is found only for a smooth cutoff whereas a sharp cutoff 
implies a nonuniversal non-exponential $L$ dependence of $\Delta \chi[10,29,33$. 


\section{Perturbative treatment of finite-size effects for $d<4$}

In this Section we present two different perturbative treatments of the finitesize effects of the lattice model (2.1) for finite $n$. We shall focus our interest on $\Delta \chi$ in the region $L \gg \xi$ above $T_{c}$ where lattice effects are expected to be non-negligible according to the exact results of the preceding Section. In particular we show that only the first version of the perturbative treatment (in Subsection 4.1) correctly predicts the exponential size-dependence of $\Delta \chi \propto e^{-L / \xi_{1}}$.

\subsection{Ordinary perturbation theory}

First we use ordinary perturbation theory with respect to $u_{0}$ without separating the lowest $(\mathbf{k}=\mathbf{0})$ mode of $\varphi(\mathbf{x})$. In one-loop order above $T_{c}$ the inverse (bare) susceptibility of the lattice model (2.1) in a cubic geometry with periodic boundary conditions is given by 10

$$
\chi^{-1}=J_{0} \xi^{-2}\left[1+4(n+2) u_{0} J_{0}^{-2} \xi^{2} \tilde{D}(\xi, L, \tilde{a})+O\left(u_{0}^{2}\right)\right]
$$

with 


$$
\tilde{D}=L^{-d} \sum_{\mathbf{k}}\left(\xi^{-2}+\hat{J}_{\mathbf{k}} / J_{0}\right)^{-1}-\int_{\mathbf{k}}\left(\xi^{-2}+\hat{J}_{\mathbf{k}} / J_{0}\right)^{-1}
$$

where $\xi$ is the second-moment correlation length. The function

$$
\tilde{D}(\xi, L, \tilde{a})=J_{0} D\left(J_{0} \xi^{-2}, L, \tilde{a}\right)
$$

can be represented in terms of Bessel functions according to (3.10) and (3.11). Eqs. (4.1) - (4.3) are valid for general $d>2$. Because of the $\mathbf{k}=\mathbf{0}$ term in the sum of (4.2) the perturbative expression (4.1) is not applicable to the region $\xi \gg L$. In this region a separation of the lowest mode from the higher modes is necessary (see Sect. 4.2). But here we are interested in the region $L \gg \xi$ where the function $\tilde{D}$ is well behaved according to (3.12). Applying the RG procedure of Ref. [10 to the bare expression (4.1) and using the asymptotic form (3.12) leads to the scaling result for $d<4$ and for $L \gg \xi_{1} \gg \tilde{a}$

$$
\Delta \chi=g\left(L / \xi_{1}\right)=4(n+2) u^{*} d\left(2 \pi L / \xi_{1}\right)^{(1-d) / 2} e^{-L / \xi_{1}}+O\left(u^{* 2}\right)
$$

where $u^{*}$ is the fixed point value of the renormalized coupling [34] and where $\xi_{1}$ is the exponential bulk correlation length given by (2.18), up to two-loop corrections. Eq. (4.4) has the same form as (3.14). It also agrees with and goes beyond our previous perturbative result (106) of Ref. [10]. Now 
we see that it is $\xi_{1}=\xi /\left[1-(\tilde{a} / \xi)^{2} / 24+\ldots\right]$ rather than $\xi$ that should be employed in the scaling representation, similar to the case $n \rightarrow \infty$ discussed in the preceding Section. Thus the interpretation of Ref. [10] in terms of a violation of finite-size scaling was incomplete since the lattice constant $\tilde{a}$ can be absorbed in $\xi_{1}$ in a natural way. The conclusions drawn in the preceding Section after (3.14) regarding the validity of finite-size scaling in terms of $\xi_{1}$ apply also to finite $n$, at least in one-loop order. (Beyond one-loop order we expect that the exponential part of (4.4) contains the bulk correlation length $\xi_{1}$ whose universal amplitude ratio $\lim _{t \rightarrow 0+} \xi_{1} / \xi$ is slightly larger than 1 [14, 16].) Clearly these conclusions can be extended to a $d$-dimensional system with partially finite geometry that is confined in $\tilde{d}$ dimensions and is infinite in $d-\tilde{d}$ dimensions. In this case the result (4.4) remains valid except that the prefactor $d$ should be replaced by $\tilde{d}$ [10]. The result of this Subsection will be extended to the case of the continuum $\varphi^{4}$ theory in a separate paper [29].

\subsection{Separation of the lowest mode}

In the following we discuss the result for $\Delta \chi$ for $L \gg \xi$ if the standard finitesize perturbation theory [19, 20, 21, 22] is used. The details of the calculation are given in Appendix C. In this approach the lowest mode is separated and treated exactly whereas the higher modes are treated perturbatively. Accordingly we decompose 


$$
\varphi_{j}=\Phi+L^{-d} \sum_{\mathbf{k} \neq \mathbf{0}} e^{i \mathbf{k} \mathbf{x}_{j}} \hat{\varphi}_{\mathbf{k}}
$$

and $H=H_{0}+H^{\prime}$ with

$$
H_{0}(\Phi)=L^{d}\left(\frac{1}{2} r_{0} \Phi^{2}+u_{0} \Phi^{4}\right)
$$

The susceptibility (3.1) is expressed as

$$
\chi=\frac{1}{n}\left\langle\Phi^{2}\right\rangle=\frac{L^{d}}{n} \int d^{n} \Phi \Phi^{2} P(\Phi)
$$

where

$$
P(\Phi)=\exp \left[-H^{e f f}(\Phi)\right] / \int d^{n} \Phi \exp \left[-H^{e f f}(\Phi)\right]
$$

is the order-parameter distribution function with the effective Hamiltonian

$$
H^{e f f}(\Phi)=H_{0}(\Phi)+\Gamma_{0}(\Phi)
$$

The present approach consists of a perturbative expansion of $\Gamma_{0}(\Phi)$ in the exponent of $P(\Phi)$ and not of an expansion of $\chi$ itself. It turns out that this 
approach does not capture the correct (exponential) size dependence of $\Delta \chi$ for $L \gg \xi$ but instead yields

$$
\Delta \chi \propto L^{-d}
$$

in any finite order of perturbation theory (see Appendix C).

The failure of this approach is due to the fact that the separation of the zero mode [19, 20, 21, 22] is inadequate in the region $L \gg \xi$. In this region the zero mode does not have a dangerous character and all modes including the $\mathbf{k}=\mathbf{0}$ mode should be treated in the same way. This argument is valid for general $d>2$ including $d>4$ (Sect. 5). The amplitude $A\left(u_{0}\right)$ of the spurious power law $\Delta \chi \propto A\left(u_{0}\right) L^{-d}$ is only partially cancelled order by order in a perturbative treatment of the higher modes but $A\left(u_{0}\right)$ remains nonzero at any finite order of perturbation theory. A complete cancellation of $A\left(u_{0}\right)$ is achieved only in an exact treatment of the $\mathbf{k} \neq \mathbf{0}$ modes as can be seen from the exact solution for $n \rightarrow \infty$ (see Eqs. (12) and (21) of Ref. [33]). These considerations are insensitive to the lattice spacing and remain valid also within the continuum $\varphi^{4}$ theory [29].

We conclude that the perturbative calculation of finite-size effects above and at $T_{c}$ requires two different approaches depending on whether $0 \leq L / \xi \lesssim$ $O(1)$ or $O(1) \lesssim L / \xi \leq \infty$. In the former case the separation of the lowest mode is appropriate. In the latter case which includes the approach to the bulk limit at fixed $T>T_{c}$ one should employ the ordinary perturbation approach of Subsection 4.1 where all modes are treated perturbatively. To 
combine the results of both approaches requires some matching in an intermediate range of $L / \xi$. The good agreement of our previous finite-size calculations [21] with highly accurate Monte-Carlo data for the three-dimensional Ising model [35] was restricted to the region $0 \leq L / \xi \lesssim O(1)$ whereas the region $O(1) \lesssim L / \xi \leq \infty$ was not investigated. The exponential size dependence in the latter region is not correctly included in the results of Ref. 211. The same criticism applies to other finite-size calculations in the literature which are based on the separation of the lowest mode. Although these exponential effects are small they are detectable and clearly distinguishable from power-law terms as has been demonstrated very recently by Monte-Carlo simulations for the magnetization of the two- and three-dimensional Ising model [26]. 


\section{$5 \quad$ Finite-size effects for $d>4$}

In this Section we extend our study of finite size effects to $d>4$ within the lattice model (2.1) for cubic geometry and periodic boundary conditions. We focus our interest on the approach of the susceptibility $\chi$ to the bulk susceptibility $\chi_{b}$ above $T_{c}$. To provide a correct description of this approach is a basic task of finite-size theory. This corresponds to the region $L \gg \xi$ where the exponential correlation length $\xi_{1}$ is expected to become an important length scale according to the results of the preceding Sections. We shall show

that, in addition to $\xi_{1}$, the second reference length [22, 27, 36] $l_{0} \sim u_{0}^{1 /(d-4)}$ associated with the higher (inhomogeneous) modes remains relevant for the large- $L$ behavior of $\chi$ and that a single-variable (lowest-mode) finite-size scaling description 119, 24] of $\chi$ fails for $L \gg \xi$.

\subsection{Exact results for $n \rightarrow \infty$}

For $\chi_{b} \gg \tilde{a}^{2}$ the inverse bulk susceptibility above $T_{c}$ for $4<d<6$ and $n \rightarrow \infty$ at fixed $u_{0} n$ is determined by

$$
\chi_{b}^{-1}=r_{0}-r_{0 c}-4 u_{0} n \chi_{b}^{-1} \int_{\mathbf{k}} \hat{J}_{\mathbf{k}}^{-2}\left\{1+O\left[(d-4)^{-1}\left(\chi_{b}^{-1} \tilde{a}^{2}\right)^{(d-4) / 2}\right]\right\}
$$

as follows from (3.3) for $L \rightarrow \infty$. From (5.1) and (3.3) we then obtain the leading relative deviation of $\chi$ from $\chi_{b}$ for $\Delta \chi \ll 1$ as 


$$
\Delta \chi \equiv \frac{\chi_{b}-\chi}{\chi_{b}}=l_{0}^{d-4} \chi_{b} J_{0}^{2} D\left(\chi_{b}^{-1}, L, \tilde{a}\right)+O\left[(\Delta \chi)^{2}\right]
$$

with the reference length 27]

$$
l_{0}=\left[\frac{4 u_{0} n}{J_{0}^{2}\left(1+4 u_{0} n \int_{\mathbf{k}} \hat{J}_{\mathbf{k}}^{-2}\right)}\right]^{1 /(d-4)}
$$

The bulk susceptibility can be expressed in terms of the second-moment correlation length $\xi$ as $\chi_{b}=J_{0}^{-1} \xi^{2}$. Using the large- $L$ behavior of $D\left(\chi_{b}^{-1}, L, \tilde{a}\right)$ according to (3.12) we obtain from (5.2) for $L \gg \xi$

$$
\Delta \chi \sim d(2 \pi)^{(1-d) / 2}\left(L / l_{0}\right)^{4-d}(L / \xi)^{-2}\left[(L / \tilde{a}) \sinh \left(\tilde{a} / \xi_{1}\right)\right]^{(d-3) / 2} e^{-L / \xi_{1}}
$$

where now the exponential bulk correlation length $\xi_{1}$, 2.18), governs the exponential size dependence, similar to the case $d<4$. In the asymptotic region $\xi \gg \tilde{a}$ we may replace $\xi$ by $\xi_{1}$ in the non-exponential part of (5.4). This yields the two-variable finite-size scaling form

$$
\Delta \chi=g\left(L / \xi_{1},\left(L / l_{0}\right)^{4-d}\right)
$$

with the exact scaling function for $L \gg \xi \gg \tilde{a}$ 


$$
g(x, y)=d(2 \pi)^{(1-d) / 2} y x^{(d-7) / 2} e^{-x}
$$

Unlike the corresponding scaling function $g(x)$ in (3.14) for $d<4$ we see that here we need two scaling variables $x=L / \xi_{1}$ and $y=\left(L / l_{0}\right)^{4-d}$. In the present context where the lowest mode plays no particular role, the second variable $y$ is associated with the higher modes and has nothing to do with the dangerous character of $u_{0}$. The present result (5.5) and (5.6) complements our previous two-variable finite-size scaling function (138) - (142) for the lattice model in Ref. 27 where $\xi$ instead of $\xi_{1}$ was employed [37. A complete description of the scaling form of $\chi$ in the entire (asymptotic) $L^{-1}-\xi^{-1}$ plane requires to incorporate in $g(x, y)$ a kind of crossover from the variables $(L / \xi, y)$ for the region $0 \leq L / \xi \lesssim O(1)$ to $\left(L / \xi_{1}, y\right)$ for the region $O(1) \lesssim L / \xi_{1} \leq \infty$.

We recall that an alternative choice of the scaling variables $(L / \xi, y)$ is $(w, y)$ where 22]

$$
w=(L / \xi)^{2} y^{-1 / 2}=t(L / \tilde{\ell})^{d / 2}, \quad \tilde{\ell}=l_{0}\left(\xi_{0} / l_{0}\right)^{4 / d} .
$$

Correspondingly the susceptibility can be represented as 22]

$$
\chi=L^{d / 2} \tilde{P}(w, y)
$$

Instead of $(w, y)$ an equivalent choice is $\left(w^{2 / d}, y\right)$ where 


$$
w^{2 / d}=L / \ell_{T}
$$

contains Binder's "thermodynamic length" $\ell_{T}$ 38 which is related to $\xi_{0}$ and $l_{0}$ as

$$
\ell_{T}=l_{0}^{(d-4) / d} \xi^{4 / d}=l_{0}^{(d-4) / d} \xi_{0}^{4 / d} t^{-2 / d}
$$

This length scale, together with $l_{0}$, plays an important role in the region $0 \leq L / \ell_{T} \lesssim O(1)$ where the dangerous character of $u_{0}$ is important (corresponding to the region between the curved lines in Fig. 1 of Ref. [22]) but $\ell_{T}$ loses its significance outside this region. In particular in the region $O(1) \lesssim L / \xi \leq \infty$ the correlation length $\xi_{1}$ (and $\xi$ ) and the reference length $l_{0}$ associated with the higher modes [22] govern the finite-size effects, as demonstrated by (5.4) - (5.6). Thus, not $\ell_{T}$ alone but $\xi_{1}$ and $l_{0}$ are indispensable for a complete description of the finite-size effects in the entire asymptotic (large $L$, small $t$ ) region for $d>4$. In the following Subsection we show that ignoring the lengths $\xi$ or $\xi_{1}$ and $l_{0}$ implies an incorrect large- $L$ dependence of $\chi$ at any fixed $T>T_{c}$.

\subsection{Lowest-mode approximation for $n \rightarrow \infty$}

Neglecting the $\mathbf{k} \neq \mathbf{0}$ contributions to $\chi$ leads to 


$$
\chi_{0}=2\left[r_{0}+\left(r_{0}^{2}+16 u_{0} n L^{-d}\right)^{1 / 2}\right]^{-1}
$$

for $n \rightarrow \infty$ at fixed $u_{0} n$. In this approximation we have $\chi_{b}^{0}=r_{0}^{-1}=\left(a_{0} t\right)^{-1}$. This yields

$$
\Delta \chi_{0} \equiv \frac{\chi_{b}^{0}-\chi_{0}}{\chi_{b}^{0}}=1-2\left[1+\left(1+16 u_{0} n r_{0}^{-2} L^{-d}\right)^{1 / 2}\right]^{-1}
$$

In the present approximation the lengths $\xi_{0}$ and $l_{0}$ are reduced to

$$
\xi_{0}=\left(J_{0} / a_{0}\right)^{1 / 2}, l_{0}=\left(4 u_{0} n J_{0}^{-2}\right)^{1 /(d-4)} .
$$

Thus $\Delta \chi_{0}$ can be expressed in terms of the thermodynamic length $\ell_{T}$, (5.10), as

$$
\begin{gathered}
\Delta \chi_{0}=1-2\left\{1+\left[1+4\left(\ell_{T} / L\right)^{-d}\right]^{1 / 2}\right\}^{-1} \\
=\left(L / \ell_{T}\right)^{-d}+O\left(L^{-2 d}\right)
\end{gathered}
$$

for $L \gg \ell_{T}$. Comparison of (5.14) with (5.4) - (5.6) shows that the lowestmode approximation [19] fails both with regard to the $L$ dependence of $\Delta \chi$ 
as well as with regard to the temperature dependence of the reference length scale $\ell_{T} \neq \xi_{1}$ in the scaling variable.

The same criticism applies to the phenomenological single-variable scaling form $\Delta \chi=f\left(L / \ell_{T}\right)$ proposed by Binder et al. [24]. The recent statement [39] that the single-variable scaling form is presumably true asymptotically for $L \rightarrow \infty$ is correct for $T=T_{c}$ (more precisely, for $L \rightarrow \infty$ at fixed finite $w)$. Furthermore, the lowest-mode result $\chi_{0}(t, L)=L^{d / 2} \tilde{P}(w, 0)$ correctly contains the limit $\chi_{b}^{0}(t)=\chi_{0}(t, \infty)$ of $\chi_{0}(t, L)$ for $L \rightarrow \infty$ at fixed $L / \xi$ (see Eq. (104) of [22] and Eq. (102) of [27]) but does not correctly describe the size dependence in approaching this limit $\chi_{b}^{0}(t)$ (see Eq. (104) of [27]). A corresponding statement is also true with regard to the magnetization below $T_{c}$ as confirmed by Monte Carlo simulations for the $d=5$ Ising model 40]. As pointed out in [40], the property

$$
\lim _{L \rightarrow \infty} \chi(t, L) / \chi_{b}(t)=1
$$

at arbitrary fixed $L / \xi$ is a nontrivial feature that is valid only for $d>4$ and that is correctly contained in the lowest-mode approximation and in the Binder et al. scaling form whereas for $d<4$ the same limit yields the function $f(L / \xi) \neq 1$ for $L / \xi<\infty$.

For any fixed $T \neq T_{c}$, however, the leading size dependence $\propto L^{-d}$ predicted by the single-variable scaling form [24] is incorrect. The origin for this defect are the missing higher modes. At fixed $w$ for large $L$, these modes only cause (slowly decaying) corrections $\sim O\left(y^{1 / 2}\right)$ to the leading size dependence 
$L^{d / 2} \tilde{P}(w, 0)$ of the lowest-mode approximation [22]. For fixed $T \neq T_{c}$ and large $L$ corresponding to $w \gg 1$ and $y \ll 1$, however, the higher modes and the lowest mode must be treated in the same way, as shown in Sect. 4.2, and the effects of the higher modes become increasingly dominant with increasing $L / \xi$ and can no longer be considered only as corrections.

More specifically, the structure of the scaling function (5.8) can be written as

$$
L^{d / 2} \tilde{P}(w, y)=L^{d / 2} \tilde{P}(w, 0)+\Delta(t, L)
$$

where $\Delta$ describes the size effect of the higher modes. The size effect $\Delta_{0}$ of the zero mode is contained in

$$
L^{d / 2} \tilde{P}(w, 0)=\chi_{b}(t)+\Delta_{0}(t, L)
$$

where $\chi_{b}(t)$ is the bulk susceptibility. The crucial point now is that for sufficiently large $L \gg \xi$ the structure of $\Delta$ becomes 33

$$
\Delta=-\Delta_{0}+O\left(e^{-L / \xi_{1}}\right)
$$

i.e., the zero-mode size dependence of $\Delta_{0} \propto L^{-d}$ is exactly cancelled by the higher-mode size dependence of $\Delta$. Thus, $\Delta$ is not small compared to $\Delta_{0}$ 
and it is inadequate to refer to the size effects of $\Delta$ only as "corrections to the lowest-mode result" [39, 41].

\subsection{Perturbative treatment for finite $n$}

For finite $n$ a perturbative treatment of the finite-size effects becomes necessary. Our arguments (in Sect. 4) for the necessity of two different perturbative approaches remain valid also for $d>4$. A one-loop perturbation calculation on the basis of a separation of the lowest mode was presented recently [22] for the case $n=1$. The results of this calculation are applicable to the region $0 \lesssim L / \xi \lesssim O(1)$ but the quality of this approach deteriorates with increasing $L / \xi$ in the region $O(1) \lesssim L / \xi \leq \infty$. For the latter region the following ordinary perturbation calculation with respect to $u_{0}$ is appropriate.

The bare perturbative one-loop expression (4.1) remains valid also for $d>4$. This leads to

$$
\Delta \chi=4(n+2) u_{0} J_{0}^{-2} \xi^{2} \tilde{D}(\xi, L, \tilde{a})+O\left(u_{0}^{2}\right)
$$

where $\tilde{D}$ is given by (4.3) and (3.10). Here we interpret the prefactor $4(n+$ 2) $u_{0} J_{0}^{-2}$ as $l_{0}^{d-4}+O\left(u_{0}^{2}\right)$ as indicated by the result (5.2) for $\chi$ in the large- $n$ limit. Using (4.3) and (3.12) for $L \gg \xi$ we arrive at the same expression for $\Delta \chi$ as given already in (5.4) where now the reference length $l_{0}$ for finite $n$ is 


$$
l_{0}=\left\{\frac{4 u_{0}(n+2)}{J_{0}^{2}\left[1+4 u_{0}(n+8) \int_{\mathbf{k}} \hat{J}_{\mathbf{k}}^{-2}\right]}\right\}^{1 /(d-4)} .
$$

The coefficient $4(n+8)$ in the denominator is inferred from the form (5.3) in the large- $n$ limit and from the previous result in (30) of Ref. 222] for $n=1$. (Our present definition of $l_{0}$ differs from that of Ref. [22] by the factor $\left.[4(n+2)]^{1 /(d-4)}\right)$. In the asymptotic region $L \gg \xi \gg \tilde{a}$ we arrive at a one-loop scaling form of $\Delta \chi$ for finite $n$ which is identical with (5.5) and (5.6). The $n$ dependence enters only the expressions for $l_{0}$ and

$$
\xi_{0}=\left(J_{0} / a_{0}\right)^{1 / 2}\left[1+4(n+2) u_{0} \int_{\mathbf{k}} \hat{J}_{\mathbf{k}}^{-2}\right]^{1 / 2}
$$

For $n=1$ this result for $\Delta \chi$ complements our previous two-variable finitesize scaling function (97) and (99) in Ref. [22] which did not yet incorporate the exponential size dependence $\propto e^{-L / \xi_{1}}$ for $L \gg \xi$.

Similar to the case $n \rightarrow \infty$, the lowest-mode approximation [19] and the phenomenological single-variable scaling form [24] lead to the incorrect power law for finite $n$

$$
\Delta \chi_{0}=\frac{4(n+2) u_{0}}{r_{0}^{2}} L^{-d}+O\left(L^{-2 d}\right)=\left(L / \ell_{T}\right)^{-d}+O\left(L^{-2 d}\right)
$$


with $\ell_{T}$ given by (5.10). Our comments after (5.14) apply also to (5.23). In summary, while the two-variable scaling form correctly embodies the $L$ dependent approach $\propto e^{-L / \xi_{1}}$ to the bulk limit at fixed $T>T_{c}$ this crucial information is lost in the single-variable scaling form and in the lowest-mode approximation. Therefore the reduction of the two scaling variables to a single scaling variable is not justified.

Very recently our prediction of the exponential size dependence (5.6) has been confirmed by Monte Carlo simulations for the five-dimensional Ising model [25, 26].

An analysis of finite-size effects for $d>4$ within the continuum $\varphi^{4}$ theory will be given in a separate paper [29] where it is shown that the finite-size effects depend significantly on the cutoff procedure. For a smooth cutoff the results for the Binder cumulant at $T_{c}$ and for the two-variable finite-size scaling function of $\chi$ are found to be different from those found previously [22, 27, 33, 36, 42] for a sharp cutoff, see also the note added in Ref. [10. 


\section{One-dimensional Ising model}

In this Section we illustrate in an elementary way the close connection between the exponential bulk correlation length $\xi_{1}$ and the finite-size scaling structure for the example of the exactly solvable one-dimensional Ising model. Although the critical temperature $T_{c}=0$ vanishes, this model has well defined correlation lengths $\xi$ and $\xi_{1} \neq \xi$ for $T>0$, which diverge for $T \rightarrow T_{c}=0$.

First we consider spins $s_{j}= \pm 1$ on sites $x_{j}$ of an infinite chain with a lattice spacing $\tilde{a}$. The Hamiltonian reads

$$
H=-J \sum_{j=-\infty}^{\infty} s_{j} s_{j_{+1}}
$$

The correlation function is well known, see e.g. Ref. [43]. The exact result has an exponential form for arbitrary distances $\left|x_{i}-x_{j}\right|$,

$$
<s_{i} s_{j}>=\exp \left(-\left|x_{i}-x_{j}\right| / \xi_{1}\right)
$$

with the exponential correlation length

$$
\xi_{1}=\tilde{a}\left[\ln \left(\lambda_{+} / \lambda_{-}\right)\right]^{-1}
$$


where $\lambda_{+}$and $\lambda_{-}$are the eigenvalues of the transfer matrix 43] with $\lambda_{+}>\lambda_{-}$. Obviously (6.2) has a scaling form in terms of $\xi_{1}$.

In order to calculate the correlation length $\xi$ as defined in (1.2) we consider the Fourier transform

$$
\hat{G}(k)=\sum_{j=-\infty}^{\infty}<s_{0} s_{j}>\exp (-i k j \tilde{a})
$$

where $s_{0}$ denotes the spin on a fixed site $x_{0}$. Using (6.2) and (6.3) we obtain

$$
\hat{G}(k)=\frac{1-\left(\lambda_{-} / \lambda_{+}\right)^{2}}{1+\left(\lambda_{-} / \lambda_{+}\right)^{2}-2\left(\lambda_{-} / \lambda_{+}\right) \cos k \tilde{a}} .
$$

This leads to the exact result $\hat{G}(0)=\exp (2 \beta \varepsilon)$ and

$$
\xi^{2}=\hat{G}(0) \frac{\partial}{\partial k^{2}}[\hat{G}(k)]_{\left.\right|_{k=0}}^{-1}=\frac{\tilde{a}^{2}}{4\left[\sinh \left(\tilde{a} / 2 \xi_{1}\right)\right]^{2}}
$$

where $\xi_{1}$ is given by (6.3). This relation between $\xi$ and $\xi_{1}$ is identical with (2.17) or (2.18) which was derived for the $\varphi^{4}$ model in the large- $n$ limit in Sect. 3 and in one-loop order in Sect. 4 . In particular we again have $\xi_{1} / \xi \rightarrow 1$ for $T \rightarrow T_{c}=0$.

Now we consider the finite-size effect on the susceptibility $\chi_{L}$ of a finite onedimensional Ising chain which consists of $N$ spins and which has a length 
$L=N \tilde{a}$. We assume periodic boundary conditions. The partition function is 43] $Z_{N}=\lambda_{+}^{N}+\lambda_{-}^{N}$ which is valid also at finite magnetic field $h$. The second derivative with respect to $h$ leads to the exact finite-size scaling form of the relative deviation from the bulk susceptibility at $h=0$

$$
\Delta \chi=\frac{\chi_{\infty}-\chi_{L}}{\chi_{\infty}}=\frac{2 e^{-L / \xi_{1}}}{1+e^{-L / \xi_{1}}} .
$$

Eq. (6.7) is valid for arbitrary $L / \xi_{1}$ where $\xi_{1}$ is identical with the exponential bulk correlation length (6.3).

The crucial point is that $L / \xi_{1}$ and not $L / \xi$ is the natural finite-size scaling variable. If $\Delta \chi$ were expressed in terms of $\xi$ then an apparent violation of finite-size scaling would arise from the $\tilde{a}$ dependent difference between $\xi_{1}$ and $\xi$

$$
\Delta \chi=2 e^{-L / \xi} \exp \left(L \tilde{a}^{2} / 24 \xi^{3}\right)
$$

for $L \gg \xi \gg \tilde{a}$, in the same way as found previously [10] for the $\varphi^{4}$ model. Similarly, the bulk scaling form for the correlation function would be violated if the result (6.2) would be expressed in terms of $\xi$. Thus the exact results (6.2), (6.6) and (6.7) demonstrate in an elementary way the significant difference between $\xi$ and $\xi_{1}$ as well as the close connection between bulk and finite-size scaling.

It would be interesting to extend this analysis to the exact results for the 
two-dimensional Ising model [44, 45] and to compare these results with the exponential size dependence found in recent Monte Carlo data in Fig. 2c of Ref. [26].

\section{Note added}

The distinction between $\xi$ and $\xi_{1}$ is significant also for resolving discrepancies in the interpretation of Monte Carlo simulation results of percolation phenomena [46].

\section{Acknowledgment}

Support by Sonderforschungsbereich 341 der Deutschen Forschungsgemeinschaft and by NASA under contract number 1201186 is acknowledged. One of us (X.S.C.) thanks the National Natural Science Foundation of China for support under Grant No. 19704005. We also thank the referees for useful comments. 


\section{Appendix A : Anisotropy of the exponential correlation length}

We start from (2.14) for the case where $\mathbf{x}=\left(x_{1}, x_{2}, 0, \ldots\right)$,

$$
G(\mathbf{x})=\frac{\tilde{a}^{2-d}}{2 J} \int_{0}^{\infty} d s e^{-(\tilde{a} / \xi)^{2} s} e^{-2 d s}\left[I_{0}(2 s)\right]^{d-2} I_{\nu_{1}}(2 s) I_{\nu_{2}}(2 s)
$$

with $\nu_{i}=\left|x_{i}\right| / \tilde{a}$. For large $\nu$ and large $s=\nu z / 2$ we have 47

$$
I_{\nu}(\nu z) \sim(2 \pi \nu)^{-1 / 2} q^{-1 / 2} \exp \left(\nu\left\{q+\ln \left[z(1+q)^{-1}\right]\right\}\right)
$$

with $q=\left(1+z^{2}\right)^{1 / 2}$. Furthermore we use the large- $s$ behavior 48

$$
I_{0}(2 s)=e^{2 s}(4 \pi s)^{-1 / 2}\left[1+O\left(s^{-1}\right)\right] .
$$

For sufficiently large $\nu_{i}$ a saddle-point approach suffices to perform the integration over $s$ and to determine the exponential large- $\left|x_{i}\right|$ behavior of (A.1). Introducing the angle $\theta$ according to $\nu_{1}=r \cos \theta, \nu_{2}=r \sin \theta$ we obtain the exponential part of $G(\mathbf{x})$ as

$$
G(\mathbf{x}) \sim \exp \left\{-|\mathbf{x}| / \xi_{1}(\theta)\right\}
$$


where $\xi_{1}(\theta)$ denotes the anisotropic exponential correlation length. The angular dependence is given by

$$
\begin{aligned}
\frac{\tilde{a}}{\xi_{1}(\theta)} & =(\cos \theta) \ln \left[u^{1 / 2} \cos \theta+\left(1+u \cos ^{2} \theta\right)^{1 / 2}\right] \\
& +(\sin \theta) \ln \left[u^{1 / 2} \sin \theta+\left(1+u \sin ^{2} \theta\right)^{1 / 2}\right]
\end{aligned}
$$

where

$$
\begin{gathered}
u=b\left(b^{2}-4\right)\left[b+\left(b^{2} \sin ^{2} 2 \theta+4 \cos ^{2} 2 \theta\right)^{1 / 2}\right]^{-1}, \\
b=2+(\tilde{a} / \xi)^{2} / 2 .
\end{gathered}
$$

For the case $\theta=0$ this yields

$$
\xi_{1}(0)=\frac{\tilde{a}}{2}\left[\operatorname{arcsinh}\left(\frac{\tilde{a}}{2 \xi}\right)\right]^{-1}
$$

corresponding to (2.18). For the case $\theta=\pi / 4$ the result is

$$
\xi_{1}(\pi / 4)=\frac{\tilde{a}}{2^{3 / 2}}\left[\operatorname{arcsinh}\left(\frac{\tilde{a}}{2^{3 / 2} \xi}\right)\right]^{-1} .
$$


Eqs. (A.8) and (A.9) agree with (4.12) and (4.14) of [13 for $d=2$. For $\xi \gg \tilde{a}$ we obtain from (A.5) - (A.7)

$$
\frac{\tilde{a}}{\xi_{1}(\theta)}=\frac{\tilde{a}}{\xi}\left[1-\frac{1}{48}\left(1+\cos ^{2} 2 \theta\right)\left(\frac{\tilde{a}}{\xi}\right)^{2}+O\left(\tilde{a}^{4} / \xi^{4}\right)\right] .
$$

For $\theta=0$ and $\theta=\pi / 4$, A.10) disagrees with (4.13) and (4.15) of Ref. [13]. Asymptotically $(\xi \rightarrow \infty)$ we obtain from (A.10) $\xi_{1} / \xi \rightarrow 1$ for all $\theta$ (in the spherical limit and in one-loop order). Nevertheless, because of the exponential form of $G(\mathbf{x})$, the nonasymptotic $\theta$-dependence remains nonnegligible in $G(\mathbf{x})$ for $|\mathbf{x}| \gtrsim 24 \xi^{3} / \tilde{a}^{2}$ even arbitrarily close to $T_{c}$, see the reasoning in the context of (2.22) and (2.23).

To derive the large- $|x|$ behavior (2.16) for the case $\mathbf{x}=(x, 0, \ldots)$ we use (A.2) and (A.3). Expanding around the maximum of the exponential part of the integrand of $C(x),(2.15)$, and performing the integration over $s$ leads to (2.16). 


\section{Appendix B : Bulk correlation function in one- loop order for $d<4$}

In terms of the second-moment correlation length (1.2) the bare bulk twopoint vertex function at finite $\mathbf{k}$ above $T_{c}$ is given in one-loop order for $d>2$ by 10

$$
\Gamma^{(2)}\left(\mathbf{k}, \xi, u_{0}, \tilde{a}, d\right)=\hat{J}_{\mathbf{k}}+J_{0} \xi^{-2}+O\left(u_{0}^{2}\right)
$$

corresponding to the integrand of $G(\mathbf{x})$ in the form of $(2.11)$ and $(2.10)$. Employing the renormalization procedure at finite lattice constant $\tilde{a}$ as described in Section 2.2 of Ref. 10 we obtain for $d \leq 4$

$$
\Gamma^{(2)}(\mathbf{k})=Z_{\varphi}^{-1}\left[\hat{J}_{\mathbf{k}}+J_{0} \xi^{-2}+O\left(u(\ell)^{2}\right)\right] \exp \int_{1}^{\ell} \zeta_{\varphi}\left(\ell^{\prime}\right) \frac{d \ell^{\prime}}{\ell^{\prime}}
$$

For the application to $T>T_{c}$ we choose the flow parameter as $\ell=\xi_{0} / \xi$ where $\xi_{0}$ is the asymptotic amplitude of $\xi$ above $T_{c}$. For the case $\mathbf{x}=(x, 0,0 \ldots)$ and in the limit $|x| \gg \tilde{a}$ the correlation function is in one-loop order for $d \leq 4$

$$
G(\mathbf{x})=\int_{\mathbf{k}}\left[\Gamma^{(2)}(\mathbf{k})\right]^{-1} e^{i \mathbf{k x}}=Z_{\varphi} C\left(x, \xi_{1}, \tilde{a}\right) \exp \int_{\ell}^{1} \zeta_{\varphi}\left(\ell^{\prime}\right) \frac{d \ell^{\prime}}{\ell^{\prime}}
$$


with $C\left(x, \xi_{1}, \tilde{a}\right)$ given by (2.16)-(2.18), apart from corrections of $O\left(u(\ell)^{2}\right)$, where $u(\ell)$ is the effective four-point coupling [10]. In the asymptotic region $\xi_{1} \gg \tilde{a}$ we obtain from (B.3)

$$
G(\mathbf{x})=Z_{\varphi}\left[A^{(2)}\right]^{-1} \xi^{-\eta} \xi_{0}^{\eta} C\left(x, \xi_{1}, \tilde{a}\right)\left[1+O\left(u^{* 2}\right)\right]
$$

with

$$
A^{(2)}=\exp \left\{\int_{1}^{0}\left[\zeta_{\varphi}\left(\ell^{\prime}\right)-\zeta_{\varphi}(0)\right] \frac{d \ell^{\prime}}{\ell^{\prime}}\right\}
$$

and the critical exponent $\eta=-\zeta_{\varphi}(0)$. In (B.4) the asymptotic $\left(|x| \gg \xi_{1}\right)$ form of $C\left(x, \xi_{1}, \tilde{a}\right)$ is

$$
C\left(x, \xi_{1}, \tilde{a}\right)=\frac{\tilde{a}^{2-d}}{4 J}\left(\frac{\tilde{a}}{2 \pi|x|}\right)^{(d-1) / 2}\left(\frac{\tilde{a}}{\xi_{1}}\right)^{(d-3) / 2} e^{-|x| / \xi_{1}} .
$$

Asymptotically we may replace $\xi^{\eta}$ in (B.4) by $\xi_{1}^{\eta}\left[1+O\left(\xi^{-2}\right)\right]$. Therefore $G(\mathbf{x})$ can be rewritten in the asymptotic scaling form for $d<4$

$$
G(\mathbf{x})=(\tilde{a} /|x|)^{d-2+\eta} \Phi\left(|x| / \xi_{1}\right)
$$

with the scaling function for $|x| / \xi_{1} \gg 1$ 


$$
\Phi\left(|x| / \xi_{1}\right)=\tilde{A} \frac{\tilde{a}^{2-d}}{4 J(2 \pi)^{(d-1) / 2}}\left(\frac{|x|}{\xi_{1}}\right)^{\frac{1}{2}(d-3)+\eta} \exp \left(-|x| / \xi_{1}\right)
$$

apart from $O\left(u^{* 2}\right)$ corrections. The amplitude $\tilde{A}$ is for $d<4$ 110

$$
\tilde{A}=Z_{\varphi}\left(u, \tilde{a} / \xi_{0}, d\right)\left(\xi_{0} / \tilde{a}\right)^{\eta}\left[A^{(2)}\right]^{-1}
$$




\section{Appendix C : Separation of the lowest mode}

In the following we show that the separation of the lowest mode implies $\Delta \chi \propto L^{-d}$ for $L \gg \xi$ and for general $d>2$ in any finite order of perturbation theory. In leading order of the $\mathbf{k} \neq \mathbf{0}$ modes the effective Hamiltonian (4.9) becomes 22

$$
\begin{gathered}
H^{e f f}(\Phi)=L^{d}\left[\frac{1}{2} r_{0}^{e f f} \Phi^{2}+u_{0}^{e f f} \Phi^{4}\right] \\
r_{0}^{e f f}=r_{0}-r_{0 c}+4(n+2) u_{0}\left[L^{-d} \sum_{\mathbf{k} \neq \mathbf{0}}\left(r_{0}-r_{0 c}+\hat{J}_{\mathbf{k}}\right)^{-1}-\int_{\mathbf{k}} \hat{J}_{\mathbf{k}}^{-1}\right] \\
u_{0}^{e f f}=u_{0}-4(n+8) u_{0}^{2} L^{-d} \sum_{\mathbf{k} \neq \mathbf{0}}\left(r_{0}-r_{0 c}+\hat{J}_{\mathbf{k}}\right)^{-2}
\end{gathered}
$$

Since we work here at finite lattice spacing we have incorporated the finite shift $r_{0 c}=-4(n+2) u_{0} \int_{\mathbf{k}} \hat{J}_{\mathbf{k}}^{-1}+O\left(u_{0}^{2}\right)$ of the parameter $r_{0}$ already at oneloop order. For simplicity we have not included here the zero-mode average $M_{0}^{2}$ defined previously [21, 22] since it is negligible in the region $T>T_{c}$. It is convenient to rewrite $r_{0}^{e f f}$ and $u_{0}^{\text {eff }}$ in terms of the second-moment bulk correlation length $\xi$. Using the bare one-loop relation [10] 


$$
r_{0}-r_{0 c}=J_{0} \xi^{-2}\left\{1+4(n+2) u_{0} \int_{\mathbf{k}}\left[\hat{J}_{\mathbf{k}}\left(\hat{J}_{\mathbf{k}}+J_{0} \xi^{-2}\right)\right]^{-1}+O\left(u_{0}^{2}\right)\right\}
$$

we obtain

$$
\begin{gathered}
r_{0}^{e f f}=J_{0} \xi^{-2}-4(n+2) u_{0} J_{0}^{-1} \Delta_{1}\left(\xi^{-2}\right), \\
u_{0}^{e f f}=u_{0}-4(n+8) u_{0}^{2} J_{0}^{-2} \int_{\mathbf{k}}\left(\xi^{-2}+\hat{J}_{\mathbf{k}} / J_{0}\right)^{-2}+4(n+8) u_{0}^{2} J_{0}^{-2} \Delta_{2}\left(\xi^{-2}\right), \\
\Delta_{m}\left(\xi^{-2}\right)=\int_{\mathbf{k}}\left(\xi^{-2}+\hat{J}_{\mathbf{k}} / J_{0}\right)^{-m}-L^{-d} \sum_{\mathbf{k} \neq \mathbf{0}}\left(\xi^{-2}+\hat{J}_{\mathbf{k}} / J_{0}\right)^{-m} .
\end{gathered}
$$

Note that, because of the separation of the zero-mode, the sums in (C.6) and (C.7) do not contain the $\mathbf{k}=\mathbf{0}$ part that was still contained in the function $\tilde{D}$, (4.2). Therefore we obtain the relation $\Delta_{1}\left(\xi^{-2}\right)=L^{-d} \xi^{2}-\tilde{D}(\xi, L, \tilde{a})$. The important consequence is that the power-law term $\propto L^{-d}$ in $\Delta_{1}\left(\xi^{-2}\right)$ becomes dominant for large $L / \xi$ compared to the exponential behavior $\propto e^{-L / \xi_{1}}$ of $\tilde{D}$ according to (4.3) and (3.12). Similarly we have the non-exponential behavior $\Delta_{2} \propto L^{-d}$ for large $L / \xi$. 
In the present approximation the susceptibility is

$$
\begin{gathered}
\chi=\frac{1}{n}\left(\frac{L^{d}}{u_{0}^{e f f}}\right)^{1 / 2} \vartheta_{2}\left(Y^{e f f}\right), \\
Y^{e f f}=L^{d / 2} r_{0}^{e f f}\left(u_{0}^{e f f}\right)^{-1 / 2}, \\
\vartheta_{2}(y)=\frac{\int_{0}^{\infty} d s s^{n+1} \exp \left(-\frac{1}{2} y s^{2}-s^{4}\right)}{\int_{0}^{\infty} d s s^{n-1} \exp \left(-\frac{1}{2} y s^{2}-s^{4}\right)} .
\end{gathered}
$$

These expressions are valid for general $d>2$ in the sense of bare perturbation theory. If we take the limit $\tilde{a} \rightarrow 0$ and apply the field-theoretic RG approach to (C.8) - C.10) we reproduce the results of Ref. [21] for $2<d<4$ which are well applicable to the critical region $0 \leq L / \xi \lesssim O(1)$. In this region lattice effects are negligible.

In the region $L / \xi \gg 1$ corresponding to $Y^{e f f} \gg 1$ the function $\vartheta_{2}$ has the asymptotic expansion 49

$$
\vartheta_{2}(y)=n y^{-1}\left[1-4(n+2) y^{-2}+O\left(y^{-4}\right)\right] \text {. }
$$

Within the lowest-mode approximation, where $r_{0}^{\text {eff }}=J_{0} \xi^{-2}$ and $u_{0}^{\text {eff }}=u_{0}$, the expansion (C.11) implies 


$$
\begin{gathered}
\chi=J_{0}^{-1} \xi^{2}\left[1-4(n+2) u_{0} J_{0}^{-2} \xi^{4} / L^{d}+O\left(L^{-2 d}\right)\right], \\
\Delta \chi=4(n+2) u_{0} J_{0}^{-2} \xi^{4} L^{-d}+O\left(L^{-2 d}\right) .
\end{gathered}
$$

We see that the lowest-mode approximation yields the incorrect (non-exponential) size dependence $\propto L^{-d}$ for large $L$. In order to see whether this defect is remedied in higher order we proceed by including the next terms of (C.5) and (C.6). Then it turns out that the $O\left(u_{0}\right)$ contribution $\propto \Delta_{1} \propto L^{-d}$ in (C.5) cancels the $O\left(u_{0}\right)$ term in (C.12) and (C.13). Therefore $\Delta \chi$ appears to become proportional to $u_{0}^{2}$ according to

$$
\begin{gathered}
\Delta \chi=-c u_{0}^{2} J_{0}^{-4} \xi^{8-d} L^{-d}+O\left(L^{-2 d}\right), \\
c=8(n+2)(n+8) A_{d}(d-2)(4-d)^{-1}, \\
A_{d}=\Gamma(3-d / 2) 2^{2-d} \pi^{-d / 2}(d-2)^{-1},
\end{gathered}
$$

for $2<d<4$. But in this order the leading size dependence $\Delta \chi \propto L^{-d}$ in (C.14) is still non-exponential. We anticipate that in the next order of this approach the $u_{0}^{2}$ term of (C.14) will also be cancelled, thus $\Delta \chi \propto u_{0}^{3} L^{-d}$, etc. We conclude that the present approach does not capture the correct (exponential) size dependence of $\Delta \chi$ for $L \gg \xi$ in any finite order of perturbation 
theory. An application of the renormalization group for $d<4$ would not remedy this defect for $L \gg \xi$. In the present context the renormalization of (C.14) would only change $J_{0}^{-2} u_{0}$ to $u^{*} \xi^{d-4}$ and this would only change the critical temperature dependence of (C.14) to $\Delta \chi=-c u^{* 2}(L / \xi)^{-d}$ for $d<4$ in the region $L \gg \xi \gg \tilde{a}$. 


\section{References}

[1] E. Brézin, J. C. Le Guillou, J. Zinn-Justin, in Phase Transitions and Critical Phenomena, edited by C. Domb and J. L. Lebowitz (Academic, New York, 1976), Vol. 6, p. 125.

[2] D. Amit, Field Theory, the Renormalization Group and Critical Phenomena (World Scientific, Singapore, 1984).

[3] J. Zinn-Justin, Quantum Field Theory and Critical Phenomena (Clarendon Press, Oxford, 1996).

[4] M. E. Fisher, in Critical Phenomena, Proceedings of the 1970 International School of Physics "Enrico Fermi", Course 51, edited by M. S. Green (Academic, New York, 1971).

[5] E. Brézin, J. Phys. (Paris) 43, 15 (1982).

[6] M. N. Barber, in Phase Transitions and Critical Phenomena, edited by C. Domb, J.L. Lebowitz (Academic Press, New Yor, 1983), Vol. 8, p. 145 .

[7] Finite Size Scaling and Numerical Simulation of Statistical Systems, edited by V. Privman (World Scientific, Singapore, 1990).

[8] K. Binder, Rep. Progr. Phys. 60, 487 (1997).

[9] M.P. Gelfand and M.E. Fisher, Physica A 166, 1 (1990), see Sect. 1.2.

[10] X.S. Chen and V. Dohm, Eur. Phys. J. B 10, 687 (1999). 
[11] V. Privman and M.E. Fisher, Phys. Rev. B 30, 322 (1984), see the paragraph after Eq. (2.5).

[12] See the paragraph after (4.2.1) of Ref. [9].

[13] M.E. Fisher and R.J. Burford, Phys. Rev. 156, 583 (1967).

[14] H.B. Tarko and M.E. Fisher, Phys. Rev. B 11, 1217 (1975).

[15] A.P. Gottlob and M. Hasenbusch, Physica A 201, 593 (1993).

[16] M. Campostrini, A. Pelissetto, P. Rossi, and E. Vicari, Phys. Rev. E 57, 184 (1998).

[17] P. Provero, Phys. Rev. E 57, 3861 (1998).

[18] M. Caselle, M. Hasenbusch, and P. Provero, Nucl. Phys. B 556 [FS], 575 (1999).

[19] E. Brézin, J. Zinn-Justin, Nucl. Phys. B 257 [FS14], 867 (1985).

[20] J. Rudnick, H. Guo, and D. Jasnow, J. Stat. Phys. 41, 353 (1985).

[21] A. Esser, V. Dohm, and X.S. Chen, Physica A 222, 355 (1995)

[22] X.S. Chen, V. Dohm, Int. J. Mod. Phys. C 9, 1073 (1998).

[23] V. Privman, M.E. Fisher, J. Stat. Phys. 33, 385 (1983).

[24] K. Binder, M. Nauenberg, V. Privman, A.P. Young, Phys. Rev. B 31, 1498 (1985).

[25] D. Stauffer, private communication. 
[26] X.S. Chen, V. Dohm, D. Stauffer, Eur. Phys. J B, in print.

[27] X.S. Chen, V. Dohm, Eur. Phys. J. B 5, 529 (1998).

[28] M. Abramowitz and I.A. Stegun, eds., Handbook of Mathematical Functions (Dover Publ., New York, 1972).

[29] X.S. Chen and V. Dohm, in preparation.

[30] P.M. Morse, H. Feshbach, Methods of Theoretical Physics (Mc GrawHill, New York, 1953)

[31] M. N. Barber, M. E. Fisher, Ann. Phys. (N.Y.) 77, 1 (1973).

[32] We find agreement with (8.7) - (8.9) of Ref. 31 provided that $\left[\Phi_{0}\left(1+\Phi_{0}\right)\right]^{(d-3) / 4}$ in (8.9) is replaced by the more complete expression $\left[\sinh \Gamma_{d}(T)\right]^{(d-3) / 2}$ according to (B.18) and (B.11) of Ref. [31], with $\Gamma_{d}(T)=2 w_{c}=2 \operatorname{ar} \sinh \left(\Phi_{0}^{1 / 2} / 2\right)$ and $\Phi_{0}=(\tilde{a} / \xi)^{2}$, see also Sect. 5.3 of Ref. [10].

[33] X.S. Chen, V. Dohm, Eur. Phys. J. B 7, 183 (1999). On the right-hand sides of equations (11), (13) and (14) the signs of the last terms have to be reversed.

[34] For the relation between $u^{*}$ and the fixed point value $u_{\min }^{*}$ of the minimally renormalized theory see the last paragraph in Sect. 4 of Ref. [10.

[35] X.S. Chen, V. Dohm, A.L. Talapov, Physica A 332, 375 (1996).

[36] X.S. Chen, V. Dohm, Physica A 251, 439 (1998). 
[37] If we replace $\xi_{1}$ by $\xi$ in (5.6) we find exact agreement between our Eq. (5.6) and Eq. (102) of Ref. [27] for $\hat{x} \gg 1$.

[38] K. Binder, Z. Phys. B 61, 13 (1985).

[39] K. Binder, E. Luijten, M. Müller, N.B. Wilding, H.W.J. Blöte, preprint.

[40] M. Cheon, I. Chang, and D. Stauffer, Int. J. Mod. Phys. C 10, 131 (1999).

[41] E. Luijten, K. Binder, and H.W.J. Blöte, Eur. Phys. J. B 9, 289 (1999).

[42] X.S. Chen, V. Dohm, Int. J. Mod. Phys. C 9, 1007 (1998); condmat/9711298.

[43] R.J. Baxter, Exactly Solved Models in Statistical Mechanics (Academic Press, London, 1982).

[44] A. E. Ferdinand, M. E. Fisher, Phys. Rev. 185, 832 (1969).

[45] H. Au-Yang, M.E. Fisher, Phys. Rev. B 11, 3469 (1975); B 21, 3956 (1980).

[46] K. Malarz and A.M. Vidales, Int. J. Mod. Phys. C 9, 147 (1998).

[47] See Eq. 9.7.7 of Ref. [28].

[48] See Eq. 9.7.1 of Ref. [28].

[49] For a representation of $\vartheta_{2}(y)$ in terms of parabolic cylinder functions see Appendix A of Ref. 21]. Their asymptotic expansion [28] leads to (C.11). 